\title{
PRÁCTICAS EDUCATIVAS PATERNAS Y AGRESIVIDAD EN ESTUDIANTES UNIVERSITARIOS
}

\author{
Ma Teresa Cerezo Rusillo \\ Pedro Félix Casanova Arias

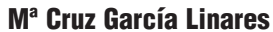 \\ $M^{\mathrm{a}}$ de la Villa Carpio Fernández \\ Departamento de Psicología, Universidad de Jaén, \\ Campus de las Lagunillas, s/n, 23071 Jaén. \\ E-mail: macerezo@ujaen.es \\ https://doi.org/10.17060/ijodaep.2017.n1.v1.899
}

Fecha de Recepción: 1 Enero 2017

Fecha de Admisión: 1 Abril 2017

\section{RESUMEN}

Este estudio examina la relación existente entre las prácticas educativas paternas y el comportamiento agresivo (agresión física, verbal, ira y hostilidad) presentado por un grupo de 437 estudiantes universitarios.

Los instrumentos utilizados fueron el Cuestionario de Agresividad (Buss y Perry, 1992) y la Escala para la evaluación del Estilo Parental (Oliva, Parra, Sánchez-Queija y López, 2007).

Los resultados muestran que el afecto, el fomento de la autonomía, la revelación y el humor manifestados por ambos progenitores, se relacionan negativamente con el comportamiento agresivo informado por los estudiantes.

Sin embargo, el control psicológico ejercido por los padres y madres se relacionó positivamente con el comportamiento agresivo.

No se encontraron relaciones entre el control conductual de padres y madres y la manifestación de comportamientos agresivos.

Palabras clave: Estilo educativo paterno, agresividad

\section{ABSTRACT}

This study analyzes the relationship between parents' educational practices and aggressive behavior (physical aggression, verbal, anger and hostility) presented by a group of 437 university students .

The instruments used were the Aggression questionnaire (Buss \& Perry, 1992) and the Parental Style Assessment Scale (Oliva, Parra, Sánchez-Queija y López, 2007). 


\section{PRÁCTICAS EDUCATIVAS PATERNAS Y AGRESIVIDAD EN ESTUDIANTES UNIVERITARIOS}

The results indicate that affection, autonomy, revelation and humor manifested by both parents, are negatively related to the aggressive behavior reported by students.

However, psychological control exercised by fathers and mothers was positively related to the aggressive behavior.

No relationship was found between the behavioral control of fathers and mothers and the manifestation of aggressive behavior.

Keywords: Paternal educational style, aggressiveness

\section{PRÁCTICAS EDUCATIVAS PATERNAS Y AGRESIVIDAD EN ESTUDIANTES UNIVERITARIOS Introducción}

En las últimas décadas ha habido un creciente interés en analizar cómo las relaciones familiares se asocian con los problemas de ajuste psicosocial que aparecen en niños y adolescentes, como es la agresividad.

Tradicionalmente el estudio de estas relaciones ha sido abordado desde una perspectiva tipológica, intentándose establecer una relación entre el comportamiento agresivo de niños y adolescentes y el estilo educativo manifestado por sus progenitores. Dicho estilo educativo ha venido siendo configurado por la consideración conjunta de las dimensiones afecto y control manifestado por los padres, estableciéndose cuatro estilos educativos: democrático, permisivo, autoritario y negligente (Baumrind, 1971; Maccoby y Martin, 1983). Teniendo en cuenta esta clasificación, numerosas investigaciones en las que se ha examinado la relación existente entre los estilos educativos paternos y el comportamiento agresivo de los hijos, han puesto de manifiesto que los estilos caracterizados por la ausencia de afecto, el escaso intercambio comunicativo o la imposición severa de normas, características todas ellas del estilo educativo parental autoritario, se relacionan en mayor medida con la aparición de comportamientos agresivos en los hijos, mientras que el estilo democrático, se relaciona con un menor grado de expresión de conducta agresiva (Aparici, 2004; Côté, Barker, Nagin y Tremblay, 2007; De la Torre-Cruz, García-Linares y Casanova-Arias, 2014; Kawabata et al., 2011; Tur-Porcar et al., 2012).

Sin embargo, tal y como indicaron Steinberg y Silk en 2002, este enfoque tipológico debe ser complementado con un enfoque de tipo dimensional, desde el cual se aborde el estudio de las relaciones que mantienen algunas de las variables 0 dimensiones que resultan más relevantes dentro de cada estilo parental, con variables referidas al ajuste psicosocial de los hijos (Oliva, Parra, Sánchez y López, 2007).

Partiendo de este enfoque dimensional, diversos estudios han explorado la relación existente entre la conducta agresiva y distintos constructos referidos a la interacción entre padres e hijos en la vida cotidiana. Uno de estos constructos es el tipo de control ejercido por los padres. En este sentido, se ha venido estableciendo un efecto diferencial de dos tipos de control parental sobre el comportamiento violento de los hijos. Así, diversas investigaciones han evidenciado que el control conductual, caracterizado por la exigencia de responsabilidades, el establecimiento de límites y la supervisión, se relaciona negativamente con la aparición de agresividad en los hijos. Sin embargo, cuando los padres utilizan métodos basados en el control psicológico, como es la inducción de culpa 0 el chantaje emocional, los hijos tienden a manifestar en mayor medida comportamientos violentos (Márquez, Villarreal, Verdugo y Montes, 2014).

Otras investigaciones han puesto de manifiesto que el excesivo tanto control conductual como psicológico 0, las prácticas educativas caracterizadas por ausencia de control, también favorecen la aparición de conductas de agresividad en los hijos (Krnutson, DeGarmo y Reid, 2004; Mazefsky y Farell, 2005).

Un aspecto relacionado con el control por parte de los padres y que cabe mencionar, es el conocimiento que éstos tienen de distintos aspectos de la vida de sus hijos. En este sentido Kerr y Stattin (2000) indican que en ocasiones, la forma de determinar el control parental ha consistido en eva- 
luar lo que los padres saben sobre el comportamiento de sus hijos. Sin embargo, lo importante no es lo que saben de sus hijos e hijas, sino los medios mediante los cuales obtienen esta información. Así, establecen tres formas: con preguntas directas, por el control explícito y por lo que los hijos e hijas cuentan espontáneamente (autorevelación). De estas tres formas de obtener información sobre los hijos, es la autorevelación la que mejor predice el ajuste adolescente. De hecho, esta estrategia ha sido considerada como una manera sutil de control parental relacionada con la prevención de la aparición de algunos comportamientos de riesgo, como es el comportamiento agresivo (Crouter, Bumpus, Davis y McHale, 2005; Oliva, 2006; Ortiz, 2015).

Por otra parte, además del tipo de control ejercido por los padres, otras prácticas educativas paternas se han asociado a la aparición de comportamientos agresivos en la infancia y adolescencia. Así, diversos estudios han puesto de manifiesto que prácticas educativas basadas en la falta de comunicación, favorecen el desarrollo de conductas agresivas por parte de los hijos, mientras que cuando los padres manifiestan afecto, promueven la autonomía y una buena comunicación, es menos probable la aparición de conductas de agresividad (Estévez, Murgui, Moreno y Musitu (2007); Senabre, Ruiz Ordónez y Murgui, 2012).

Teniendo en cuenta todo lo expuesto, el presente trabajo pretende profundizar en el estudio de las relaciones existentes entre las prácticas educativas parentales y el comportamiento agresivo de los hijos, ya que, aunque existe evidencia empírica acerca la de existencia de dichas relaciones en etapas como la infancia y la adolescencia, son escasos los estudios que abordan esta temática en edades más tardías.

\section{MÉTODO}

\section{Participantes}

La muestra estuvo compuesta por 437 estudiantes de distintas titulaciones impartidas en la Facultad de Humanidades y Ciencias de la Educación de la Universidad de Jaén, que de manera voluntaria participaron en el estudio. Treinta y dos eran estudiantes del Grado en Historia del arte, 154 estudiaban Psicología, 45 eran alumnos del Grado en estudios ingleses, 78 eran alumnos del grado en Educación Infantil y 71 del Grado en Educación Primaria. Cincuenta y siete estudiantes no especificaron la titulación en la que estaban cursando sus estudios.

De los participantes 101 (23.12\%) eran hombres y 336 (76.88\%) mujeres y sus edades oscilaban entre 18 y 43 años, siendo la edad media de 19,9 años.

\section{Instrumentos}

Estilo Parental (Oliva, Parra, Sánchez-Queija y López Gaviño, 2007).

Es una escala compuesta por 41 ítems que evalúa la percepción que el adolescente tiene del estilo parental de sus progenitores. El sujeto debe responder a 41 ítems para la madre y a otros 41 para el padre, utilizando para ello una escala de puntuaciones comprendidas entre 1 y 5 ( $1=$ Nunca, $2=$ Pocas Veces, $3=$ Algunas Veces, $4=$ A menudo y $5=$ Siempre) .

Esta escala se divide a su vez en 6 sub-escalas: afecto y comunicación (8 ítems), siendo el coeficiente de fiabilidad Alfa de Cronbach para el madre de .88 y el padre de .90; promoción de autonomía (8 ítems), siendo el coeficiente de fiabilidad alfa $=.83 / .83$; control conductual ( 6 ítems), siendo su fiabilidad de alfa = .76/.78; control psicológico (8 ítems), siendo su coeficiente de fiabilidad alfa de .80/.80; revelación (5 ítems), cuya fiabilidad es de .83/.85) y humor (6 ítems), cuya fiabilidad es de.82/.82.

Versión del Aggressive Questionnaire (Buss y Perry, 1992).

Es un cuestionario utilizado como herramienta para evaluar el comportamiento agresivo en la adolescencia. En este estudio se empleó la versión de 29 Ítems la cual presenta una estructura fac- 
torial de cuatro dimensiones: Agresividad física (p.e.: "Si alguien me golpea, le respondo golpeándole también"), Agresividad verbal (p.e.: "He amenazado a gente que conozco"), Ira (p.e.: "Cuando estoy frustrado muestro el enfado que tengo") y Hostilidad (p.e.: "A veces soy bastante envidioso") que aportan información de los diferentes aspectos cognitivos (pensamientos negativos, resentimiento, desconfianza), comportamentales (agresión física y verbal) y afectivo- emocionales (rabia, cólera o repulsa) de la agresividad. El cuestionario presenta un formato tipo Likert con cinco opciones de respuesta (Siendo $1=$ completamente falso, $2=$ bastante falso, $3=$ ni verdadero ni falso, $4=$ bastante verdadero y $5=$ completamente verdadero) donde el encuestado debe seleccionar una sola opción en función de si el contenido del ítem se adecua en mayor o menor medida a su comportamiento. Una elevada puntuación se corresponde con un mayor comportamiento agresivo y hostil. Los índices de fiabilidad calculados mediante el coeficiente alpha de Cronbach es de .86 para agresividad física, .71 para agresividad verbal, .67 para ira y .76 para hostilidad. Estos valores son similares a los hallados por Andreu, Peña y Graña (2002).

\section{RESULTADOS}

Para conocer las relaciones existentes entre las prácticas educativas de ambos progenitores y la agresividad que presentan los hijos se realizó un análisis de correlación a través del estadístico de Pearson.

En cuanto a las prácticas educativas paternas (tabla 1), los resultados muestran la existencia de una relación negativa entre la manifestación de afecto, la presencia de sentido del humor, la revelación y el control psicológico ejercido por el padre y la agresión tanto física como verbal y la hostilidad informada por los estudiantes. Además se obtuvieron relaciones positivas entre esos mismos indicadores de la agresividad y el control psicológico paterno. También este tipo de control se relacionó de manera positiva con la expresión de ira por parte de los hijos.

Tabla 1. Correlaciones entre las prácticas educativas paternas y comportamientos agresivos de los hijos

\begin{tabular}{|c|c|c|c|c|c|c|c|}
\hline & & $\begin{array}{c}\text { AFECTO } \\
\text { Y } \\
\text { COMUNICACIÓN }\end{array}$ & $\begin{array}{c}\text { CONTROL } \\
\text { CONDUCTUAL }\end{array}$ & AUTONOMIA & $\begin{array}{c}\text { CONTROL } \\
\text { PSICOLÓGICO }\end{array}$ & REVELACIÓN & HUMOR \\
\hline \multirow[t]{3}{*}{$\begin{array}{l}\text { AGRESIÓN } \\
\text { FísICA }\end{array}$} & $\begin{array}{l}\text { Correlación } \\
\text { de Pearson }\end{array}$ &,$- 106^{2}$ & .021 &,- 092 & $.247^{\circ+}$ & $-.097^{\circ}$ & $-.104^{\circ}$ \\
\hline & $\begin{array}{l}\text { Sig. } \\
\text { (bilateral) }\end{array}$ & .031 &, 666 & .061 & .000 & .048 &, 034 \\
\hline & $\mathbf{N}$ & 416 & 419 & 419 & 419 & 419 & 419 \\
\hline \multirow[t]{3}{*}{$\begin{array}{l}\text { AGRESIÓN } \\
\text { VERBAL }\end{array}$} & $\begin{array}{l}\text { Correlación } \\
\text { de Pearson }\end{array}$ & $-103^{2}$ & 028 &,- 077 & $206^{20}$ &,$- 126^{24}$ & $-.121^{\circ}$ \\
\hline & $\begin{array}{l}\text { Sig. } \\
\text { (bilateral) }\end{array}$ & 035 & .562 &, 113 & .000 & 010 &, 013 \\
\hline & $\mathrm{N}$ & 420 & 423 & 423 & 422 & 423 & 423 \\
\hline \multirow[t]{3}{*}{ HOSTILIDAD } & $\begin{array}{l}\text { Correlación } \\
\text { de Pearson }\end{array}$ &,$- 190^{27}$ &, 042 &,$- 165^{\circ 1}$ & $285^{21}$ &,$- 120^{\circ}$ & $-.213^{11}$ \\
\hline & $\begin{array}{l}\text { Sig. } \\
\text { (bilateral) }\end{array}$ &, 000 & 391 & ,001 & .000 & .014 &, 000 \\
\hline & $\mathrm{N}$ & 416 & 419 & 419 & 418 & 419 & 419 \\
\hline \multirow[t]{3}{*}{ IRA } & $\begin{array}{l}\text { Correlación } \\
\text { de Pearson }\end{array}$ & -058 & .077 &,- 066 & $.272^{12}$ &,- 042 & .083 \\
\hline & $\begin{array}{l}\text { Sig. } \\
\text { (bilateral) }\end{array}$ & .232 & .113 & .175 & .000 & 389 &, 088 \\
\hline & $\mathrm{N}$ & 421 & 424 & 424 & 423 & 424 & 424 \\
\hline
\end{tabular}


En cuanto a la promoción de la autonomía por parte del padre solamente se encontró relación negativa con la manifestación de hostilidad en los jóvenes.

Respecto a las prácticas educativas maternas (tabla 2) el análisis muestra que el afecto y el sentido del humor y el control psicológico materno se relacionan negativamente con la presencia de agresión física y verbal y la hostilidad de los hijos. En este caso además se encontró que estas prácticas educativas maternas también se relacionaban de manera negativa con la expresión de ira por parte de los jóvenes. Sin embargo el control psicológico se relacionó positivamente con los cuatro indicadores de agresividad.

Tabla 2. Correlaciones entre las prácticas educativas maternas y comportamientos agresivos de los hijos

\begin{tabular}{|c|c|c|c|c|c|c|c|}
\hline & & $\begin{array}{c}\text { AFECTO } \\
\text { Y } \\
\text { COMUNICACIÓN }\end{array}$ & $\begin{array}{c}\text { CONTROL } \\
\text { CONDUCTUAL }\end{array}$ & AUTONOMIA & $\begin{array}{c}\text { CONTROL } \\
\text { PSICOLÓGICO }\end{array}$ & REVELACIÓN & HUMOR \\
\hline \multirow[t]{3}{*}{$\begin{array}{l}\text { AGRESIÓN } \\
\text { FISICA }\end{array}$} & $\begin{array}{l}\text { Correlación } \\
\text { de Pearson }\end{array}$ & $-169^{n+}$ &.,- 019 & $-.122^{\circ}$ & $.243^{*+}$ & $-164^{n}$ & $-.162^{+1}$ \\
\hline & $\begin{array}{l}\text { Sig. } \\
\text { (bilateral) }\end{array}$ & .000 & .693 & 011 & 000 & .001 & .001 \\
\hline & $\mathrm{N}$ & 429 & 431 & 429 & 430 & 431 & 430 \\
\hline \multirow[t]{3}{*}{$\begin{array}{l}\text { AGRESIÓN } \\
\text { VERBAL. }\end{array}$} & $\begin{array}{l}\text { Correlación } \\
\text { de Pearson }\end{array}$ &,$- 098^{2}$ & .015 &,$- 096^{*}$ & $.177^{+1}$ &,- 093 &,$- 111^{\circ}$ \\
\hline & $\begin{array}{l}\text { Sig. } \\
\text { (bilateral) }\end{array}$ & .041 & .756 &, 045 &, 000 & 052 &, 021 \\
\hline & $\mathrm{N}$ & 433 & 435 & 433 & 433 & 435 & 434 \\
\hline \multirow[t]{3}{*}{ HOSTILIDAD } & $\begin{array}{l}\text { Correlación } \\
\text { de Pearson }\end{array}$ & $.163^{n}$ & .074 &,$- 150^{2+}$ & $.255^{21}$ &,- 082 &,- 226 \\
\hline & $\begin{array}{l}\text { Sig. } \\
\text { (bilateral) }\end{array}$ & .001 & .125 & .002 & .000 & 090 & .000 \\
\hline & $\mathrm{N}$ & 428 & 430 & 428 & 428 & 430 & 429 \\
\hline \multirow[t]{3}{*}{ IRA } & $\begin{array}{l}\text { Correlación } \\
\text { de Pearson }\end{array}$ & $-.097^{\circ}$ & .028 &,- 079 & $.213^{\circ}$ &,- 037 &,$- 145^{\prime \prime}$ \\
\hline & $\begin{array}{l}\text { Sig. } \\
\text { (bilateral) }\end{array}$ & .043 & 562 & 098 & .000 & .445 & .002 \\
\hline & $\mathrm{N}$ & 435 & 437 & 435 & 435 & 437 & 436 \\
\hline
\end{tabular}

En cuanto a la promoción de la autonomía, en el caso de la madre, se relacionó negativamente con todas las manifestaciones de agresividad, excepto con la ira, relacionándose también la revelación de manera negativa con la agresividad física de los hijos.

Por último, se pudo observar una ausencia de relación entre el control conductual ejercido por ambos progenitores y la manifestación de comportamientos agresivos en los hijos.

\section{DISCUSIÓN}

Tal y como ha quedado reflejado en investigaciones anteriores, el estilo de educativo utilizado por los padres está muy relacionado con la aparición de conductas de agresión por parte de los hijos. Los resultados de esta investigación parecen avalar la hipótesis de que hay determinadas dimensiones dentro de cada estilo educativo que se relacionan con la aparición de algún tipo de conducta violenta por parte de los hijos. Como ya obtuvieron Barber y Harmon (2002), se ha encontrado que existe una correlación positiva entre el psicológico ejercicio tanto por el padre como por la 


\section{PRÁCTICAS EDUCATIVAS PATERNAS Y AGRESIVIDAD EN ESTUDIANTES UNIVERITARIOS}

madre y la aparición en los hijos de conductas violentas. Concretamente se ha encontrado que el control psicológico ejercido por parte de ambos progenitores, se asocia a la presencia de agresión física, agresión verbal, ira y hostilidad en los jóvenes. Estos resultados son congruentes con los encontrados en diversos estudios realizados con niños y adolescentes en los que se ha relacionado este tipo de control con la manifestación de violencia (Márquez, Villarreal, Verdugo y Montes, 2014; ). Por lo tanto, parece ser que, esta forma de control basado en el chantaje y la inducción de sentimientos de culpabilidad, sigue ejerciendo sus efectos incluso después de esas edades.

Sin embargo, en lo referente al control conductual, en contra de lo encontrado en etapas anteriores, en este estudio se ha podido observar que a los inicios de la etapa adulta, no se asocia a la presencia de comportamientos agresivos. Esto podría deberse a que, en estas edades, los jóvenes precisan en menor medida de este tipo de control por parte de sus padres, por lo que éstos no suelen ejercer tal control sobre los hijos. De todas formas, este aspecto deberá ser analizado con mayor profundidad en investigaciones posteriores.

Además, de los resultados se desprende que, tal y como viene ocurriendo en la niñez y adolescencia (Estévez, Murgui, Moreno y Musitu (2007) ; Senabre, Ruiz Ordónez y Murgui, 2012) en la etapa adulta temprana, algunas prácticas educativas positivas como el establecimiento de una adecuada comunicación, la expresión de afecto hacia los hijos y el humor manifestado por los padres, se relacionan con una menor manifestación de conductas violentas en los jóvenes (Ortiz, 2015).

En cuanto a la promoción de la autonomía y la autorevelación, los resultados muestran la existencia de una relación negativa con la agresión informada por los jóvenes, siendo dicha relación distinta en ambos progenitores. Así, mientras que la promoción de autonomía se relaciona disminución de comportamientos violentos, sobre todo cuando es la madre la que la ejerce, la revelación se relaciona con una menor manifestación dichos comportamientos, sobre todo cuando es el padre el confidente.

Aunque todo lo expuesto nos indica que, a pesar de los cambios en las relaciones entre padres e hijos con el tiempo, la familia continúa constituyendo una importante influencia para el desarrollo y el ajuste en edades posteriores, este estudio no está exento de limitaciones. En primer lugar, el diseño utilizado ha sido transversal, por lo que no es posible establecer relaciones causales entre las distintas variables estudiadas. Por este motivo y con el fin de profundizar más sobre las relaciones existentes entre las prácticas educativas de padres y madres y la agresividad manifestada por lo hijos, sería interesante plantear un estudio longitudinal. Además, las conductas agresivas se han evaluado mediante medidas de autoinforme, siendo más conveniente utilizar la información aportada por otros agentes (padres, iguales, etc.), ya que, al ser estas conductas censurables, los participantes podrían estar falseando sus respuestas y minimizando este tipo de comportamientos.

\section{REFERENCIAS}

Andreu, J.M., Peña, M.E., y Graña, J.L. (2002). Adaptación psicométrica de la versión española del Cuestionario de Agresión [Psychometric adaptation of the spanish version of the Aggression Questionnaire]. Psicothema, 14, 476-482.

Aparici, G. (2004). Estilos de crianza y variables escolares relacionados con la conducta prosocial y la conducta agresiva. Universitat de València.

Barber, B. K., y Harmon, E. L. (2002). Violating the self: parental psycho-logical control of children and adolescents. En B. K. Barber (Ed.), Intru-sive parenting: how psychological control affects children and adolescents (pp. 15-52). Washington: American Psychological Association.

Baumrind, D. (1971). Current patterns of parental authority. Developmental Psychology, 4(1), 1-103. Buss, A.H., y Perry, M. (1992). The aggression questionnaire. Journal of Personality and Social Psychology, 63, 452-459 
Côté, S., Vaillancourt, T., Barker, E. D., Nagin, D. y Tremblay, R. E. (2007). The joint development of physical and indirect aggression: Predictors of continuity and change during childhood. Development and Psychopatology, 19, 37-55.

Crouter, A. C., Bumpus, M. F., Davis, K. D. y Mchale, S. M. (2005). How do parents learn about adolescents' experiences? Implications for parental knowledge and adolescent risky behavior. Child Development, 76, 869-882.

De la Torre-Cruz, Manuel J.; García-Linares, M. C.; Casanova-Arias, P.F. (2014). Relaciones entre estilos educativos parentales y agresividad en adolescentes. Electronic Journal of Research in Educational Psychology, vol. 12, núm. 32, pp. 147-170

Estévez, E. - Murgui, S. - Moreno, D. - Musitu, G. (2007). "Estilos de comunicación familiar, actitud hacia la autoridad institucional y conducta violenta del adolescente en la escuela". Psicothema, 19(1), 108-113.

Kawabata, Y., Alink, L. R. A., Tseng, W., van IJzendoorn, M. H. y Crick, N.R. (2011). Maternal and paternal parenting styles associated with relational aggression in children and adolescents: A conceptual analysis and meta-analytic review. Developmental Review, 31, 240-278.

Krnutson, J.F., DeGarmo, D.S. y Reid, J.B. (2004). Social Disadvantage and Neglectful Parenting as Precursors to the Development of Antisocial and Aggressive Child Behavior: Testing a Theoretical Model. Aggressive Behavior, 30, 187-205

Maccoby, E. E. y Martin, J. A. (1983). Socialization in the context of the family: Parent-child interaction. En García Linares, M.C., Carpio Fernández, M.V., De la Torre, M.J.; García, M.C.; Cerezo, M.T. y Casanova, P.F (2006). Estilos educativos paternos, rendimiento y violencia escolar (pg283-291). Psicología del Aprendizaje. Edit. PSICOEX.

Márquez González, C. V., Villarreal Caballero, L., Verdugo Lucero, J. C., y Montes Delgado, R. (2014). Control psicológico parental y violencia escolar entre adolescentes en una muestra mexicana.

Mazefsky, C.A. y Farell, A.D. (2005). The role of witnessing violence, peer provocation, family support and parenting practices in the aggressive behavior of rural adolescents. Journal of child and family studies, 14(1), 71-85

Oliva, A. (2006). Relaciones familiares y desarrollo adolescente. Anuario de Psicología, 37, 209-224.

Oliva, A., Parra, A., Sánchez Queija, I. y López, F. (2007). Estilos Educativos Materno y Paterno: Evaluación y relación con el ajuste adolescente. Anales de psicología, 23(1), 49-56.

Ortiz Gálvez, A. A. (2015). Prácticas educativas paternas y agresión. Trabajo Fin de Grado. Universidad de Jaén.

Senabre, P., Ruiz, Y. y Murgui, S. (2012). Estilos de parentalidad y su relación con la conducta agresiva. Edetania, 42, 145-157.

Steinberg, I., y Silk, J. S. (2002). Parenting adolescents. En I. Bornstein (Ed.), Handbook of parenting, Vol. I. Children and parenting (pp. 103-134). Mahwah, NJ: Lawrence Erlbaum Associates.

Tur-Porcar, A., Mestre, V., Samper, P. y Malonda, E. (2012). Crianza y agresividad de los menores: ¿es diferente la influencia del padre y de la madre? Psicothema, 24, 284-288. 
